\title{
Detection of Myocardial Injury Using miRNAs Expression as Genetic Biomarkers in Acute Cardiac Care
}

\author{
Alexandru Florin Rogobete ${ }^{1,2}$, Ovidiu Horea Bedreag ${ }^{1,2}$, Sonia Elena Popovici ${ }^{1}$, Adriana Mariana \\ Sas $^{1}$, Adrian Tudor Stan ${ }^{3}$, Emil Robert Stoicescu ${ }^{1}$, Dorel Sandesc ${ }^{1,2}$ \\ ${ }^{1}$ Faculty of Medicine, "Victor Babeș" University of Medicine and Pharmacy, Timișoara, Romania \\ ${ }^{2}$ Clinic of Anesthesia and Intensive Care, "Pius Brînzeu" County Emergency Hospital, Timișoara, Romania \\ ${ }^{3}$ Faculty of Dental Medicine, "Victor Babeș" University of Medicine and Pharmacy, Timișoara, Romania
}

\section{ABSTRACT}

Cardiovascular disease is a leading cause of death globally. At present, there are many ways to diagnose this pathophysiology. The greatest disadvantages related to current biomarkers are their low specificity, low selectivity and low accuracy. A new method, extensively studied recently, is the expression of miRNAs, used as genetic biomarkers for the early diagnosis of cardiovascular diseases. This paper presents an update of miRNAs species expression that can serve as early diagnostic biomarkers and for the continuous monitoring of patients with cardiovascular disease.

Keywords: microRNA, acute myocardial infarction, acute cardiac care

\section{ARTICLE HISTORY}

Received: 10 September, 2016 Accepted: 15 October, 2016

\section{CORRESPONDENCE}

Ovidiu Horea Bedreag

P-ța Eftimie Murgu nr. 2

300041 Timișoara, Romania

Tel: +40256204400

E-mail: bedreag.ovidiu@umft.ro

\section{BACKGROUND}

Cardiovascular disease is one of the leading causes of death globally. An important reason for the increased rates of morbidity and mortality in these patients is the late diagnosis of complicated cases that progress to cardiovascular emergencies. ${ }^{1-3}$ In acute cardiac settings, a number of methods are used currently for evaluation based on biochemical-humoral biomarkers, such as cardiac troponin (TNC), creatine kinase-myocardial band isoform (CK-MB) and brain natriuretic peptide (BNP).4-7
Unfortunately, these biomarkers show low selectivity and specificity, and can often display false positive results due to their association with multiple pathophysiologies. A potential future biomarker, intensively studied lately, is the genetic expression of miRNAs. Essential features that make it the ideal candidate for use as a biomarker are its high selectivity and specificity. Moreover, investigation methods for miRNAs are noninvasive and inexpensive. ${ }^{8-10}$

In this update paper, genetic and epigenetic modifications of miRNAs in cardiovascular emergencies are pre- 
sented, together with several miRNAs that can serve as biomarkers for early diagnosis of cardiac pathologies in acute settings.

\section{BIOGENESIS AND BIOCHEMICAL CHARACTERISTICS OF mIRNAS}

miRNA biosynthesis takes place in the cell nucleus. ${ }^{11}$ The mechanisms that lead to the formation of miRNAs begin with the attack of RNA polymerase II on specific genes (Figure 1). Following the transcription reactions, the first pri-miRNAs are formed. ${ }^{12}$ After these events, the RNase II endonuclease, also known as Drosha, attacks the primiRNAs species together with the DiGeorge Syndrome Critical Region 8 (DGCR8) enzymatic cofactor, leading to the formation of pre-miRNAs. ${ }^{13,14}$ Pre-miRNAs are transported from the nucleus into the cytoplasm by coupling with the Exportin 5 transporter protein. Inside the cytoplasm, the pre-miRNAs are combined with RNase III endonuclease, also known as Dicer, resulting in mature miRNAs. The RNA-binding trans-activator protein cofactor (TRBP) is involved in the formation reactions of mature miRNAs, which will be embedded in the RNA-induced silencing complex (RISC) for its transport outside the cell. ${ }^{15}$

The miRNA species formed in this manner are exported from the cells into the extracellular space in different configurations, such as exosomes, microvesicles, or apoptotic bodies. $^{16}$

\section{MIRNA EXPRESSION IN MYOCARDIAL INJURY FOLLOWING ACUTE MYOCARDIAL INFARCTION AND REPERFUSION SYNDROME}

Ischemic heart disease is a leading cause of death among patients with cardiovascular disease. A pathophysiological effect secondary to ischemic heart disease is the inflammatory effect induced by ischemia and reperfusion syndrome (IR), which occur in cases of acute myocardial infarction immediately after repermeabilization of the acutely occluded coronary artery. ${ }^{17,18}$ Yao et al. (2016) showed significant changes in miRNA-26 in patients with IR. Moreover, they reported that miRNA-26 caused inhibition of the high mobility group box 1 (HMGB1), thus significantly reducing IR. ${ }^{19}$ Wu et al. (2016) identified an alteration of the expression of miRNA-613 in cases of IR syndrome. ${ }^{20}$ Gidlöf et al. (2013) conducted a similar study on the expression of miRNAs in patients with IR syndrome or with ST elevation myocardial infarction (STEMI). They reported a rapid increase in miRNA-1, miRNA-133a and miRNA208b. Regarding miRNA changes in patients with STEMI,

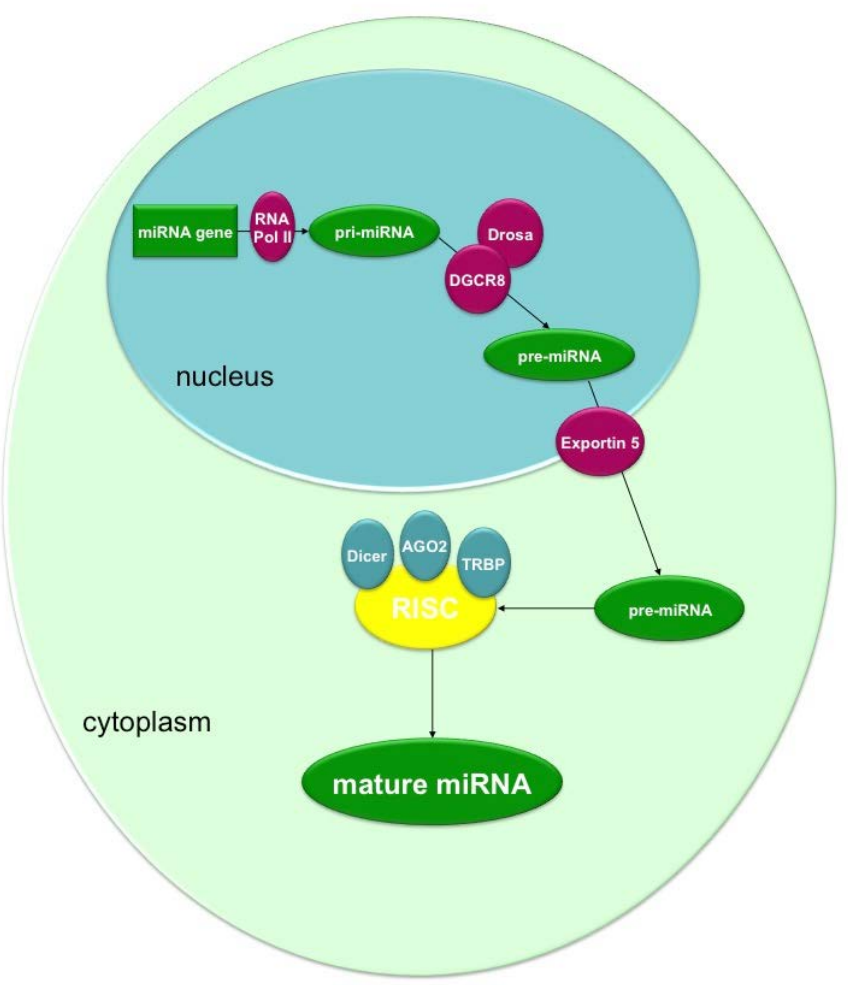

FIGURE 1. Biogenesis mechanism for miRNAs. The synthesis of miRNAs begins in the nucleus with the action of RNA polymerase II on protein-coding. This forms a first species, called pri-miRNA. Through successive reactions of polyadenylation catalyzed by DGCR8 and Drosa, the precursor for the miRNAs species, called premiRNA, is obtained. pre-miRNA thus formed is transported into the cytoplasm through Exportin 5. In the cytoplasm, the Dicer complex acts on the pre-miRNA. Subsequently, through the action of TRBP, $\mathrm{AGO} 2$ and Dicer, the RNA-induced silencing complex (RISC) and, finally, the mature miRNAs are obtained. Adapted with permission from Papurica et al. ${ }^{17}$

an increase of miRNA-1 and miRNA-133a was observed. ${ }^{21} \mathrm{~A}$ similar study on the expression of miRNAs in patients with STEMI was conducted by D'Alessandra et al. (2010), who reported an altered expression of miRNA-1, miRNA-133a, miRNA-499-5p, miRNA-122 and miRNA- $375 .{ }^{22}$

\section{MIRNA EXPRESSION IN ACUTE HEART FAILURE AND CARDIAC HYPERTROPHY}

Vegter et al. (2016) studied the expression of miRNAs in acute heart failure. The study showed statistically significant changes for several miRNAs. Moreover, it was reported that there was a number of statistical correlations between the altered expression of miRNAs and the clinical outcome. Furthermore, they identified changes in the expression of miRNA-16-5p, miRNA-106a-5p, miRNA-223-3p, miRNA-652-3p, miRNA-199a-3p and miRNA-18a-5p. ${ }^{23}$ 
Another common cardiac pathophysiology is cardiac hypertrophy. From a pathophysiological point of view, cardiac hypertrophy is an important compensatory mechanism that initially aims to reduce cardiac wall stress, but also to preserve and ensure contractile performance. Unfortunately, once it becomes chronic, this pathology leads to severe cardiac dysfunction that can rapidly deteriorate and progress to acute cardiac failure. Recent studies have reported several changes in the expression of miRNAs in the case of this pathology, which can serve as a biomarker for an early diagnosis. These included miRNA-133, miRNA-1, miRNA-208, miRNA-21, miRNA-18b, miRNA-195 and miRNA-199, miRNA-23, miRNA-24, miRNA-27 and miRNA-9. ${ }^{24}$

\section{MIRNA EXPRESSION IN CORONARY ARTERY DISEASE AND SUDDEN CARDIAC DEATH}

Coronary artery disease (CAD) is a cardiac pathology frequently associated with sudden cardiac death. Li et al. (2016), in a study on the expression of miRNAs in cases of $\mathrm{CAD}$, reported significant growth for miRNA-126-5p. ${ }^{25} \mathrm{~A}$ similar study was conducted by Liu et al. (2016), who identified changes in the expression of miRNA-208a and miRNA-370 in patients with CAD. ${ }^{26}$ Mehta et al. (2016) also reported a decrease in the expression of miRNA-132 and an increase in miRNA-143 expression in patients with CAD. ${ }^{27}$ A similar study was conducted by Sun et al. (2012), who identified a significant decrease of miRNA-126, strongly correlated statistically with the decreased concentration of low-density lipoprotein (LDL) cholesterol in patients with CAD. ${ }^{28}$ Fichtlscherer et al. (2010) reported a decrease in the expression of miRNA-17, miRNA-92a miRNA-126, miRNA-145, and miRNA-155 in patients with CAD. ${ }^{29}$

\section{PANELS OF MIRNA EXPRESSION IN ACUTE MYOCARDIAL INFARCTION}

One of the most common syndromes associated with CAD is acute myocardial infarction (AMI). In practice, the bestknown biomarker for AMI is cardiac troponin I (cTnI). A major disadvantage of cTnI is the false positives that can occur in patients with atrial fibrillation, chronic kidney disease or septic shock. Wang et al. (2013), in a study on the changes of miRNA expression in patients with AMI, showed a significant increase for miRNA-133a. ${ }^{30}$ Wang et al. (2010) also reported an increase for the expression of miRNA-1, miRNA-133a, miRNA-208a and miRNA-499 in patients with AMI. ${ }^{31} \mathrm{~A}$ similar study conducted by Cheng et al. (2010) highlights an increase in the expression of miR-
NA-1 in patients with AMI. ${ }^{32}$ Meder et al. have also studied the expression of miRNAs in the case of AMI, reporting an increase of miRNA-30c and miRNA-145. Moreover, they identified a decrease in the expression of miRNA-663b and miRNA-1291 in the case of AMI.. ${ }^{33}$

\section{CONCLUSION}

Cardiovascular diseases represent one of the leading causes of death. Early evaluation and monitoring at this moment shows no increased specificity and selectivity. An ideal biomarker for evaluating these patients may be genetic expression of miRNAs. In a conducted study, we can say that certain species of miRNAs can bring important answers about the pathophysiology of the heart. However, further studies are needed to strengthen a panel of miRNAs with expressions of high accuracy specific for cardiac pathophysiologies.

\section{CONFLICT OF INTEREST}

Nothing to declare.

\section{REFERENCES}

1. Oddo M, Rossetti AO. Predicting neurological outcome after cardiac arrest. Curr Opin Crit Care. 2011;17:254-259. doi: 10.1097/MCC.ob013e328344f2ae.

2. Zakkar M, Ascione R, James AF, Angelini GD, Suleiman MS. Inflammation, oxidative stress and postoperative atrial fibrillation in cardiac surgery. Pharmacol Ther. 2015;154:1320. doi: 10.1016/j.pharmthera.2015.06.009.

3. Doehner W, Haehling S Von, Pschowski R, Storm C, Schroeder $\mathrm{T}$. Influence of core body temperature on Tryptophan metabolism, kynurenines, and estimated IDO activity in critically ill patients receiving target temperature management following cardiac arrest. Resuscitation. 2016;107:107-114. doi: 10.1016/j.resuscitation.2016.07.239.

4. Yao X, Carlson D, Sun Y, et al. Mitochondrial ROS induces cardiac inflammation via a pathway through mtDNA damage in a pneumonia-related sepsis model. PLoS One. 2015;10:1-28. doi: 10.1371/journal.pone.0139416.

5. Tao X, Lu L, Xu Q Li S, Lin M. Cardioprotective effects of anesthetic preconditioning in rats with ischemia-reperfusion injury: propofol versus isoflurane. J Zhejiang Univ Sci B. 2009;10:740-747. doi: 10.1631/jzus.Bo920119.

6. Moore E, Bellomo R, Nichol A. Biomarkers of acute kidney injury in anesthesia, intensive care and major surgery: From the bench to clinical research to clinical practice. Minerva Anestesiol. 2010;76:425-440.

7. Devaux Y, Stammet P, Friberg $\mathrm{H}$, et al. MicroRNAs: new biomarkers and therapeutic targets after cardiac arrest? Crit Care. 2015;19:54. doi: 10.1186/s13054-015-0767-2.

8. Bryant RJ, Pawlowski T, Catto JWF, et al. Changes in circulating microRNA levels associated with prostate cancer. Br J Cancer. 2012;106:768-774. doi: 10.1038/bjc.2011.595. 
9. Pipan V, Zorc M, Kunej T. MicroRNA polymorphisms in cancer: A literature analysis. Cancers (Basel). 2015;7:1806-1814. doi: 10.3390/cancers7030863.

10. Stather PW, Sylvius N, Wild JB, Choke E, Sayers RD, Bown MJ. Differential MicroRNA expression profiles in peripheral arterial disease. Circ Cardiovasc Genet. 2013;6:490-497. doi: 10.1161/CIRCGENETICS.111.000053.

11. Bedreag $\mathrm{OH}$, Papurica $\mathrm{M}$, Rogobete $\mathrm{AF}$, et al. Using Circulating miRNAs as Biomarkers for the Evaluation and Monitoring of the Mitochondrial Damage in the Critically Ill Polytrauma Patients. Clin Lab. 2016;8:1-7. doi: 10.7754/Clin. Lab.2016.160121.

12. Abdelmohsen K, Srikantan S, Kang M-J, Gorospe M. Regulation of senescence by microRNA biogenesis factors. Ageing Res Rev. 2012;11:491-500. doi: 10.1016/j.arr.2012.01.003.

13. Papurica M, Rogobete AF, Sandesc D, et al. The Expression of Nuclear Transcription Factor Kappa B (NF-KB) in the Case of Critically Ill Polytrauma Patients with Sepsis and Its Interactions with microRNAs. Biochem Genet. 2016;54:337347. doi: 10.1007/s10528-016-9727-z.

14. Bedreag $\mathrm{OH}$, Rogobete $\mathrm{AF}$, Cradigati $\mathrm{CA}$, et al. A novel evaluation of microvascular damage in critically ill polytrauma patients by using circulating microRNAs. Rev Romana Med Lab. 2016;24:21-30. DOI:10.1515/rrlm-2016-0015

15. Bedreag OH, Sandesc D, Chiriac SD, et al. The Use of Circulating miRNAs as Biomarkers for Oxidative Stress in Critically Ill Polytrauma Patients. Clin Lab. 2016;62:263-274.

16. Dumache $\mathrm{R}$, Rogobete $\mathrm{AF}$, Bedreag $\mathrm{OH}$, et al. Use of miRNAs as Biomarkers in Sepsis. Anal Cell Pathol (Amst). 2015;2015:186716. doi: 10.1155/2015/186716.

17. Papurica M, Rogobete AF, Sandesc D, et al. Redox Changes Induced by General Anesthesia in Critically Ill Patients with Multiple Traumas. Molecular Biology International. 2015;2015:238586. doi: 10.1155/2015/238586.

18. Papurica M, Sandesc D, Rogobete AF, et al. Cardioprotective Effects Induced by Preconditioning with Halogenated Anesthetics. Journal of Interdisciplinary Medicine. 2016;1:2331. doi: 10.1515/jim-2016-0006.

19. Yao L, Lv X, Wang X. MicroRNA 26a inhibits HMGB1 expression and attenuates cardiac ischemia-reperfusion injury. J Pharmacol Sci. 2016;131:6-12. doi: 10.1016/j.jphs.2015.07.023.

20. Wu Z, Qi Y, Guo Z, Li P, Zhou D. miR-613 suppresses ischemia-reperfusion-induced cardiomyocyte apoptosis by targeting the programmed cell death 10 gene. Biosci Trends. 2016;10:251-257. doi: 10.5582/bst.2016.01122.

21. Gidlöf O, Smith JG, Miyazu K, et al. Circulating cardioenriched microRNAs are associated with long-term prognosis following myocardial infarction. BMC Cardiovasc Disord. 2013;13:12. doi: 10.1186/1471-2261-13-12.

22. D'Alessandra Y, Devanna P, Limana F, et al. Circulating microRNAs are new and sensitive biomarkers of myocardial infarction. Eur Heart J. 2010;31:2765-2773. doi: 10.1016/j. clinbiochem.2012.04.013

23. Vegter EL, Schmitter D, Hagemeijer Y, et al. Use of biomarkers to establish potential role and function of circulating microRNAs in acute heart failure. Int J Cardiol. 2016;224:231239. doi: 10.1016/j.ijcard.2016.09.010.

24. Dong D, Yang B. Role of microRNAs in cardiac hypertrophy, myocardial fibrosis and heart failure. Acta Pharm Sin B. 2011;1:1-7. doi: 10.1002/iub.204.

25. Li HY, Zhao X, Liu YZ, et al. Plasma MicroRNA-126-5p is Associated with the Complexity and Severity of Coronary Artery Disease in Patients with Stable Angina Pectoris. Cell Physiol Biochem. 2016;39:837-846. doi: 10.1159/000447794.

26. Liu H, Yang N, Fei Z, et al. Analysis of plasma miR-208a and miR-370 expression levels for early diagnosis of coronary artery disease. Biomed Rep. 2016;5:332-336. doi: 10.3892/ br.2016.726.

27. Mehta R, Otgonsuren M, Younoszai Z, Allawi H, Raybuck B, Younossi Z. Circulating miRNA in patients with nonalcoholic fatty liver disease and coronary artery disease. BMJ Open Gastroenterol. 2016;3:e000096. doi: 10.1136/ bmjgast-2016-000096.

28. Sun $X$, Zhang M, Sanagawa A, et al. Circulating microRNA-126 in patients with coronary artery disease: correlation with LDL cholesterol. Thromb J. 2012;10:16. doi: 10.1186/1477-9560-10-16.

29. Fichtlscherer S, De Rosa S, Fox H, et al. Circulating microRNAs in patients with coronary artery disease. Circ Res. 2010;107:677-684. doi: 10.1161/CIRCRESAHA.109.215566.

30. Wang F, Long G, Zhao C, et al. Plasma microRNA-133a is a new marker for both acute myocardial infarction and underlying coronary artery stenosis. J Transl Med. 2013;11:222. doi: 10.1186/1479-5876-11-222.

31. Wang GK, Zhu JQ Zhang JT, et al. Circulating microRNA: a novel potential biomarker for early diagnosis of acute myocardial infarction in humans. Eur Heart J. 2010;31(6):659666. doi: 10.1093/eurheartj/ehq013.

32. Cheng Y, Tan N, Yang J, et al. A translational study of circulating cell-free microRNA-1 in acute myocardial infarction. Clin Sci (Lond). 2010;119:87-95. doi: 10.1042/CS20090645.

33. Meder B, Keller A, Vogel B, et al. MicroRNA signatures in total peripheral blood as novel biomarkers for acute myocardial infarction. Basic Res Cardiol. 2011;106:13-23. doi: 10.1007/ s00395-010-0123-2. 